\title{
The case of a liver-transplant recipient with severe acute respiratory syndrome coronavirus 2 infection who had a favorable outcome
}

\author{
Kazuhiko Hayashi ${ }^{1}$ (1) $\cdot$ Yuki Ito $^{1} \cdot$ Ryosuke Yamane $^{1} \cdot$ Michiyo Yoshizaki $^{1} \cdot$ Komei Matsushita $^{1} \cdot$ Go Kajikawa $^{1}$. \\ Takashi Kozawa $^{1} \cdot$ Taro Mizutani $^{1} \cdot$ Yuko Shimizu ${ }^{1} \cdot$ Kenichi Nagano $^{1} \cdot$ Kosuke Tachi $^{1} \cdot$ Kentaro Yoshioka $^{1}$. \\ Hidemi Goto ${ }^{1}$
}

Received: 18 January 2021 / Accepted: 22 February 2021 / Published online: 6 March 2021

(c) Japanese Society of Gastroenterology 2021

\begin{abstract}
The outbreak of coronavirus disease 2019 (COVID-19), caused by severe acute respiratory syndrome coronavirus 2 (SARSCoV-2), was identified in 2019; thereafter, the COVID-19 outbreak became a health emergency of international concern. The impact of COVID-19 on liver-transplant recipients is unclear. Thus, it is currently unknown whether liver-transplant recipients are at a higher risk of developing complications related to COVID-19. Here, we report the case of liver-transplant recipients who were infected with SARS-CoV-2. A 20-year-old man who had undergone living-donor liver transplantation from his father at 5 years of age because of congenital biliary atresia was referred to our hospital for SARS-CoV-2 infection. Chest computed tomography did not show any abnormalities; however, laboratory results revealed liver dysfunction. He received tacrolimus as maintenance therapy that was continued at the same dose. He has not developed severe pulmonary disease and was discharged after 10 days of hospitalization. Limited data are available on post-transplant patients with COVID-19, and this case of a young patient without metabolic comorbidities did not show any association of severe COVID-19 under tacrolimus treatment. The progression of COVID-19 in liver-transplant recipients is complex, and COVID-19 risk should be evaluated in each patient until the establishment of optimal guidelines.
\end{abstract}

Keywords Severe acute respiratory syndrome coronavirus 2 - Coronavirus disease 2019 - Liver transplantation

\section{Introduction}

The outbreak of coronavirus disease 2019 (COVID-19), caused by severe acute respiratory syndrome coronavirus 2 (SARS-CoV-2), was identified in Wuhan, China in December 2019; the first COVID-19 patient in Japan was diagnosed in January 2020 [1-3]. The COVID-19 pandemic has spread to Japan and become a health emergency of national concern. The outcomes of COVID-19 are wide ranging, from asymptomatic infection to death $[4,5]$. This variability seems to exist in the clinical progression of COVID-19 between individuals, suggesting host differences. The experience with prior infectious virus outbreaks suggested that

Kazuhiko Hayashi

k-hayashi@meijohosp.com

1 Department of Gastroenterology and Hepatology, Federation of National Public Service Personnel Mutual Aid Associations Meijo Hospital, 1-3-1 Sannomaru, Naka-ku, Nagoya 460-0001, Japan the progression of disease would appear to be more rapid and severe in immunocompromised hosts. However, there is limited information on the impact of COVID-19 in immunocompromised hosts, particularly liver-transplant recipients. Therefore, many studies have been performed to understand the implication of SARS-CoV-2 infection in liver-transplant recipients. D'Antiga reported that liver-transplant recipients are not at an increased risk of COVID-19 as compared to the general population [6]. Liver injury has been reported in COVID-19 patients; however, the characteristics in patients with COVID-19-related liver damage remain unknown for liver-transplant recipients $[7,8]$. Thus, it is currently unknown whether liver-transplant recipients have increased susceptibility to the complications of COVID-19, including liver injury. The reported infection rate and mortality attributed to COVID-19 in Japan are low, and these results suggest racial differences in COVID-19 pathology [9]. Limited data are available regarding the exact effects of COVID-19 on disease progression and liver function in liver-transplant recipients; thus, further clinical information on this subject 
is necessary. Here, we report the case of a liver-transplant recipient who was infected with SARS-CoV-2 in Japan.

\section{Case report}

A 20-year-old man who had undergone living-donor liver transplantation from his father at 5 years of age because of congenital biliary atresia had high body temperature, rhinorrhea, cough, and mild fatigue. He took an over-the-counter medicine for common cold. On the next day, he visited a family clinic and was diagnosed with SARS-CoV-2 infection using real-time polymerase chain reaction (PCR). Six of his workplace colleagues were also diagnosed with SARSCoV-2 infection at the same time. Two days thereafter, he was admitted to our hospital. On admission, he complained of rhinorrhea and cough, with a body temperature of $36.4{ }^{\circ} \mathrm{C}$, a pulse rate of $74 \mathrm{bpm}$, and oxygen saturation of $98 \%$ while breathing ambient air. Chest computed tomography did not show any abnormalities; however, laboratory results revealed liver dysfunction. The clinical course is shown in Fig. 1. He received maintenance immunosuppressive therapy with tacrolimus (4 mg twice daily) for post-transplant management and ursodeoxycholic acid (200 mg twice daily) for hepatoprotective effects. We considered discontinuation or dose reduction of tacrolimus; however, we decided to continue administering the same dose, because his general condition was stable. He has not developed clinical pulmonary disease and has not required antiviral medications or oxygen supplementation. On day 6 after admission, his liver function improved and the trough tacrolimus level was $4.96 \mathrm{ng} /$ $\mathrm{mL}$. He was discharged after 10 days of hospitalization and was recommended to be in self-isolation for at least 14 days.
Thirty days after his discharge, he visited the outpatient clinic; he was negative for SARS-CoV-2 infection on realtime PCR, and his liver function had improved.

\section{Discussion}

This paper reports the case of a liver-transplant recipient who was infected with SARS-CoV-2 infection and demonstrated a favorable outcome. With the rapid global spread of COVID-19, reliable evidence is necessary to reach a consensus on COVID-19 treatment in organ transplant recipients including those with liver transplants. Several systematic reviews have summarized several aspects of COVID-19 in transplant recipients of solid organs such liver, kidney, heart, and lung [10-12]. Despite the selection and publication biases, these studies have indicated that organ transplant recipients with COVID-19 are at high risk for all major adverse outcomes. Previous reports have suggested that liver-transplant recipients are not at an increased risk of severe pulmonary disease than the general population [6, 13]. Our result also suggests that SARS-CoV-2 infection had a limited impact on liver-transplant recipients. However, liver-transplant recipients under maintenance immunosuppressive therapy are considered to be at a higher risk of severe disease of COVID-19 [14, 15]. This remains controversial, and a comparison of COVID-19 outcomes between transplanted and non-transplanted patients is needed to clarify this issue. The progression of COVID-19 may depend on the condition of liver-transplant recipients. Long-term liver-transplant recipients with metabolic comorbidities appear to have poor outcomes. Thus, $>10$ years after transplantation, male sex, age $>65$ years, obesity, hypertension,
Fig. 1 Clinical course of patient. AST aspartate aminotransferase, $A L T$ alanine aminotransferase, $A L P$ alanine alkaline phosphatase, $G G T$ gamma-glutamyl transferase, SARS-CoV-2 severe acute respiratory syndrome coronavirus 2

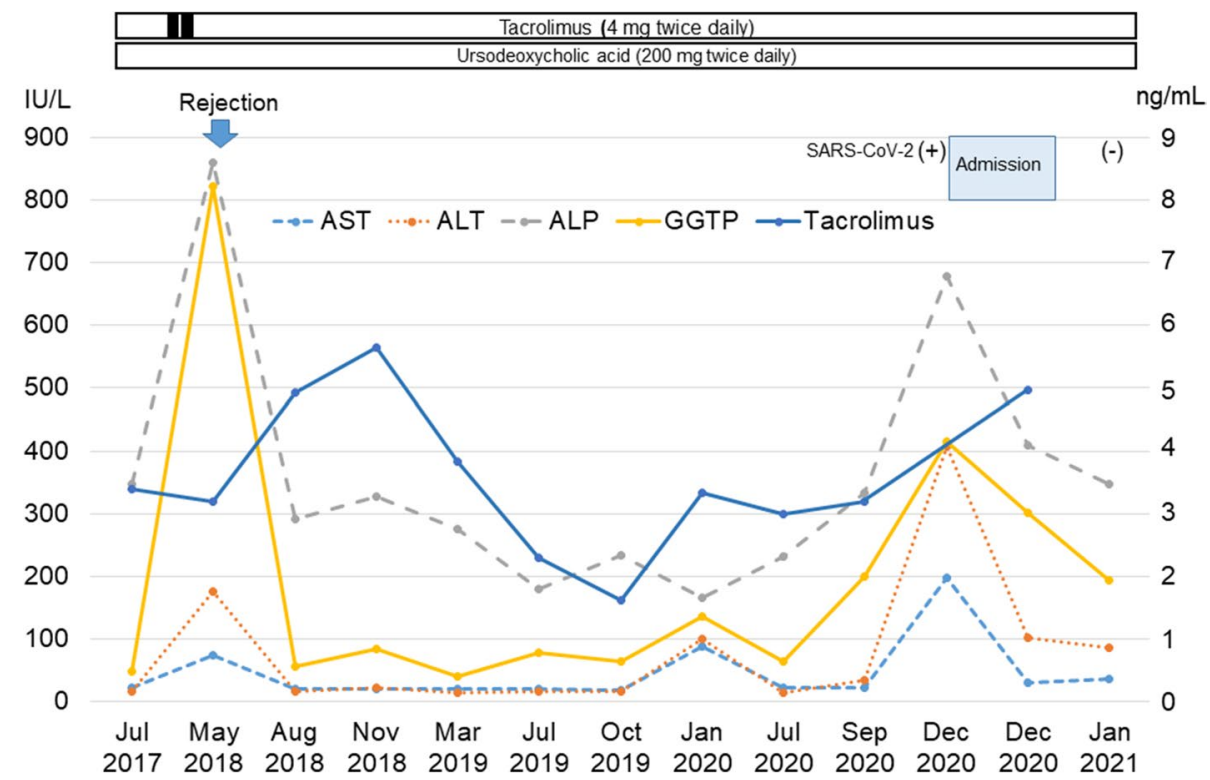


and diabetes might be major risk factors for mortality [13, 16]. In addition, a clinical history of cancer was associated with poor outcomes [17]. Our patient was a young man without complicating diseases but with the two risk factors of male sex, and a history of transplantation (that was performed 15 years previously). The clinical characteristics of liver-transplant recipients from Europe included a median age of 65 years at diagnosis and a median time of 6 years from transplantation to COVID-19 diagnosis [17]. Patients who had undergone liver transplantation a long time ago tended to be older in this cohort, although our patient had undergone liver transplantation 15 years earlier and was only 20 years old at the time of SARS-CoV-2 infection. The pediatric patients appeared to have a less severe clinical course of COVID-19 than adults $[18,19]$. Young age would overcome the impact of long duration after transplantation. Thus, the background of the liver-transplant recipients are complex, and the risk factors associated with COVID-19 prognosis should be evaluated carefully for each individual patient.

Liver injury is one of the most common clinical features of COVID-19. Liver injury appears common in patients with more severe SARS-CoV-2 infection and is associated with a higher mortality rate [20]. The risks for liver injury were related to the COVID-19 severity, history of liver disease, older age, and male sex [21]. This patient did not develop severe COVID-19 but presented with liver injury. Contrary to that in previous reports, his liver injury was not associated with disease severity. The mechanisms of hepatic impairment were speculated as the direct effect of the virus, immune reactions, hypoxia, secondary nosocomial infection, or drug-induced liver injury [21, 22]. Specific therapy for COVID-19 and oxygen therapy were not required in our patient despite the tacrolimus treatment, and an over-thecounter medicine for common cold was sufficient. Hypoxia and drug-induced liver injury could be eliminated in the current case. The same dose of tacrolimus was continued, and his tacrolimus trough level was $4.96 \mathrm{ng} / \mathrm{mL}$ on day 6 after admission. This immune condition with sufficient tacrolimus level might not cause graft rejection. The lack of liver biopsy and liver imaging studies was because he isolated to avoid secondary infection prevented the definitive diagnosis of liver dysfunction. Thinking terms of process of elimination, we speculate that the liver dysfunction might have been due to the direct effect of the virus in the present patient. The pathogenesis of liver injury with SARS-CoV-2 infection remains unclear, and further investigation on this subject is warranted.

The management of immunosuppressive drugs is a major concern in liver-transplant recipients infected with SARS-CoV-2. The effect of COVID-19 and immunosuppressive drugs on liver-transplant recipients remains unclear. Immunosuppressive drugs are not discontinued in most liver-transplant recipients with COVID-19 infection [17].
Several reports have hypothesized that immunosuppressive drugs may prevent virus replication and protect from the viral-induced cytokine storm that causes severe COVID-19 disease $[6,23]$. However, adjustment of immunosuppressive drugs might have changed the fatal outcome by secondary nosocomial infection (15). We considered discontinuation or dose reduction of tacrolimus to prevent the risk of secondary infection. This patient had an incidence of liver dysfunction 2.5 years ago and was suspected to have graft rejection due to poor compliance of tacrolimus. We believe that he had a high risk of rejection and were concerned that insufficient dose of tacrolimus might lead to graft rejection, as observed previously. The effect of immunosuppression on COVID-19 progression would be dependent on the severity of COVID19 disease and the phase of graft rejection. Considering individual risks, we decided to reduce the dose of tacrolimus but not discontinue it when he developed clinical pulmonary disease. He has not developed clinical pulmonary disease, and his liver function was improved without adjustment in the dose of tacrolimus. Insufficient immunosuppression might cause graft rejection, while excessive immune suppression can lead to nosocomial infections. The strategy for COVID19 treatment should be decided to consider the balance the risks of graft rejection and nosocomial infections.

Although the relatively small sample size and the bias in patient selection prevent us from drawing definitive conclusion about the impact of SARS-CoV-2 infection in livertransplant recipients, in this 20-year-old man, history of liver transplantation performed 15 years earlier and the absence of metabolic comorbidities were not associated with severe pulmonary disease with SARS-CoV-2 infection under tacrolimus treatment. The effects of SARS-CoV-2 infection on liver-transplant recipients are not well established, and the establishment of optimal guidelines would require time. Liver-transplant recipients must practice frequent hand washing, mask wearing, and social distancing to prevent SARS-CoV-2 infection. The progression of COVID-19 in liver-transplant recipients would is complex, and the risk of severe COVID-19 should be carefully evaluated in individual patients for optimal treatment strategies.

Acknowledgements All people declare that they have nothing to disclose. The authors thank The Meijo Hospital COVID-19 task committee, Ms. Eri Fujioka, Ms. Kaori Kikyo, and Ms. Nahomi Mito, for their efforts in COVID-19 protocol development of nursing care, and nurses and staff who worked for the safety and well-being of our patients.

\section{Declarations}

Conflict of interest Kazuhiko Hayashi, MD, PhD, Yuki Ito MD, Ryosuke Yamane MD, Michiyo Yoshizaki MD, Komei Matsushita MD, Go Kajikawa MD, Takashi Kozawa MD, Taro Mizutani MD, PhD, Yuko Shimizu MD, Kenichi Nagano MD, PhD, Kosuke Tachi MD, $\mathrm{PhD}$, Kentaro Yoshioka, MD, PhD, and Hidemi Goto, MD, PhD declare that they have no conflict of interest. 
Human rights All procedures followed have been performed in accordance with the ethical standards laid down in the 1964 Declaration of Helsinki and its later amendments.

Informed consent Informed consent was obtained from all patients for being included in the study.

\section{References}

1. Zhu N, Zhang D, Wang W, et al. A novel coronavirus from patients with pneumonia in China, 2019. N Engl J Med. 2020;382:727-33.

2. Wang C, Horby PW, Hayden FG, et al. A novel coronavirus outbreak of global health concern. Lancet. 2020;395:470-3.

3. Watanabe M. The COVID-19 pandemic in Japan. Surg Today. 2020;50:787-93.

4. Liu Y, Yan LM, Wan L, et al. Viral dynamics in mild and severe cases of COVID-19. Lancet Infect Dis. 2020;20:656-7.

5. Yang X, Yu Y, Xu J, et al. Clinical course and outcomes of critically ill patients with SARS-CoV-2 pneumonia in Wuhan, China: a single-centered, retrospective, observational study. Lancet Respir Med. 2020;8:475-81.

6. D'Antiga L. Coronaviruses and immunosuppressed patients: the facts during the third epidemic. Liver Transplant. 2020;26:832-4.

7. Xu L, Liu J, Lu M, et al. Liver injury during highly pathogenic human coronavirus infections. Liver Int. 2020;40:998-1004.

8. Fan Z, Chen L, Li J, et al. Clinical features of COVID-19-related liver functional abnormality. Clin Gastroenterol Hepatol. 2020;18:1561-6.

9. Iwasaki A, Grubaugh ND. Why does Japan have so few cases of COVID-19? EMBO Mol Med. 2020;12:e12481.

10. Marinaki S, Tsiakas S, Korogiannou M, et al. A systematic review of COVID-19 infection in kidney transplant recipients: a universal effort to preserve patients' lives and allografts. J Clin Med. 2020;9:2986.

11. Azzi Y, Bartash R, Scalea J, et al. COVID-19 and solid organ transplantation: a review article. Transplantation. 2021;105:37-55.

12. Mahalingasivam V, Craik A, Tomlinson LA, et al. A systematic review of COVID-19 and kidney transplantation. Kidney Int Rep. 2021;6:24-45.
13. Bhoori S, Rossi RE, Citterio D, et al. COVID-19 in long-term liver transplant patients: preliminary experience from an Italian Transplant Centre in Lombardy. Lancet Gastroenterol Hepatol. 2020;5:532-3.

14. Fix OK, Hameed B, Fontana RJ, et al. Clinical best practice advice for hepatology and liver transplant providers during the COVID19 pandemic: AASLD expert panel consensus statement. Hepatology. 2020;72:287-304.

15. Huang JF, Zheng KI, George J, et al. Fatal outcome in a liver transplant recipient with COVID-19. Am J Transplant. 2020;20:1907-10.

16. Webb GJ, Moon AM, Barnes E, et al. Determining risk factors for mortality in liver transplant patients with COVID-19. Lancet Gastroenterol Hepatol. 2020;5:643-4.

17. Becchetti C, Zambelli MF, Pasulo L, et al. COVID-19 in an international European liver transplant recipient cohort. Gut. 2020;69:1832-40.

18. Lu X, Zhang L, Du H, et al. SARS-CoV-2 infection in children. N Engl J Med. 2020;382:1663-5.

19. Morand A, Roquelaure B, Colson P, et al. Child with liver transplant recovers from COVID-19 infection. A case report. Arch Pediatr. 2020;27:275-6.

20. Ali N, Khaled H. Liver injury in severe COVID-19 infection: current insights and challenges. Expert Rev Gastroenterol Hepatol. 2020;14:879-84.

21. Alqahtani SA, Schattenberg JM. Liver injury in COVID-19: the current evidence. United Eur Gastroenterol J. 2020;8:509-19.

22. Jothimani D, Venugopal R, Abedin MF, et al. COVID-19 and the liver. J Hepatol. 2020;73:1231-40.

23. Carbajo-Lozoya J, Ma M, Kallies S, et al. Replication of human coronaviruses SARS-COV, HCOV-NL63 AND HCOV-229e is inhibited by the drug FK506. Virus Res. 2012;165:112-7.

Publisher's Note Springer Nature remains neutral with regard to jurisdictional claims in published maps and institutional affiliations. 